\title{
WorkingAge: Providing Occupational Safety through Pervasive Sensing and Data Driven Behavior Modeling
}

\author{
Mariagrazia Fugini, Alessandro Barenghi, Sara Comai, Gerardo Pelosi, Roberto Tedesco \\ DEIB, Politecnico di Milano, Italy. E-mail: name.surname@polimi.it
}

Marteyn van Gasteren, Carlos Alberto Catalina

ITCL Institute of Technology, Burgos, Spain. E-mail: name.surname@itcl.es

Estefanía Arribas Leal, Raquel Losada Durán, Rosa Maria Martins de Almeida

Intras, Valladolid, Spain.E-mail: name.surname@intras.es

Alexander Mertens, Vera Rick

Institute of Industrial Engineering and Ergonomics, RWTH Aachen University, Germany.

E-mail:a.mertens@iaw.rwth-aachen.de,v.rick@iaw.rwth-aachen.de

Hatice Gunes, Tian Xu

Computer Laboratory, University of Cambridge,UK. E-mail: name.surname@cl.cam.ac.uk

Gianluca Borghini, Vincenzo Ronca

BrainSigns srl, Rome, Italy.E-mail: name.surname@brainsigns.com

Hesam Sagha

audEERING GmbH, Germany. E-mail: hsagha@audeering.com

The aging of the working population calls for innovative approaches to monitor and support the changes of physical, physiological and psycho-social capabilities of workers over time, as well as to promote habits aimed at improving both health and productivity. This paper presents the WorkingAge (Smart Working Environments for All Ages - WA) project, which focuses on innovative Human Computer Interaction methods (such as augmented and/or virtual reality or gesture/voice/noise recognition or gaze tracking) to improve the users' psychological/emotional/health state at workplaces. Based on Internet of Things (IoT) technologies and on data driven models of the users' characteristics and behaviors, the WA Tool will monitor the state of users to automatically provide a set of suggestions promoting healthy habits in their working environment as well as in their daily living activities. The paper presents general concepts at the basis of the WA project, its hardware-software sensor architecture, and the elements of the ontology capturing the key concepts of the data collected to profile the users.

Keywords: Occupational Safety for Elderly, Occupational Ontology, Health at Workplaces

\section{Introduction}

Life satisfaction is directly related to the subject's socio-economic condition, health and age [De Vroom and Øverbye (2017); Grosch and Scholl (2020)]. Promoting a healthy lifestyle on both inside and outside the workplace spurs people to fulfill their potential and continuously improve their quality of life. While working is generally good for physical and mental health and well-being [Dikkers et al. (2017); Goh et al. (2015)], many workers still face adverse working conditions, due to unhealthy work environments, wrong posture and interaction with machinery, or environmental factors inducing stress such as noise or smoke. Demographic factors, such as the ageing and shrinking of the work forces [TeschRömer and Wahl (2017)], bring along progressively worse working conditions due to the induced, staff shortages and knowledge loss [Surendran (2017)], resulting in important economic and social consequences [Akkermans et al. (2016)]. New working habits should be created and supervised, so that working in diverse employment conditions does not adversely effect health, especially for ageing workers. Individual and societal interventions are needed to create desirable living

Proceedings of the 30th European Safety and Reliability Conference and the 15th Probabilistic Safety Assessment and Management Conference. Edited by Piero Baraldi, Francesco Di Maio and Enrico Zio Copyright (C) 2020 by ESREL 2020 PSAM 15 Organizers. Published by Research Publishing, Singapore ISBN: 981-973-0000-00-0 :: doi: 10.3850/981-973-0000-00-0_output 
conditions, which are characterized by autonomy and quality of life [Fitzpatrick and Moore (2018)].

This paper describes the approach to healthy ageing at work proposed in the EU funded WorkingAge (WA) project. The aim of WA is to create a hardware and software platform to enhance users' life at work, attenuating the impact of ageing on their autonomy, health and well-being. WA detects factors detrimental to a healthy life at work relying on IoT sensors in the working environment. In particular, environmental data, such as noise, are collected and combined with behavioural observations recorded via gesture/voice recognition and gaze tracking, to measure and monitor users' psychologi$\mathrm{cal} /$ emotional/health state. A Decision Support System (DSS) is developed, providing advices for workers based on their work behaviours via innovative Human Computer Interaction (HCI) methods, including augmented reality, virtual reality and so on. The DSS is based on an occupational safety ontology [Teimourikia and Fugini (2017)], to suggest corrective and preventive actions in the workplace and daily activities that may lead to an increased level of the safety, comfort and wellbeing of the employees. The main objectives of the WA project are as follows.

- Creation of a database collecting the physiological, sociological, and psychological data describing the conditions of employers and the relationships with the working environment.

- Creation of a schema including risks and challenges concerning different work settings as a function of ageing factors.

- Definition of the causes for the long-term stress of employees and its impact on the sick leave requests as well as on times off from work for vacation or on retirement times.

- Definition of a set of artificial intelligence rules to automate the creation of suggestions to ageing workers.

- Definition of a set of tips and alerts aiming at supporting the independent living for aging people to improve and maintain their productivity, functional capacity, health status as well as to preserve their physical, cognitive, psychological and social well-being.

These objectives are pursued via a User-Centered development methodology based on User Data modeling and on the design of innovative algorithms to assess stress and strain levels of users by monitoring their gesture, face, eyes, voice, and other neurometrics-related data.

The paper is organized as follows. Section 2 details the ambition, the approach and the hardware/software architecture of the WA tool. Section 3 describes the ergonomics, stress and strain assessment methodologies. Section 4 tackles how the monitoring and analysis of environmental noise in the working place is performed. Section 5] describes data acquisition and analysis of multi-modal physiological and behavioral signals. Section 6 presents the WA approach to secure data management. Section 7 outlines the designed occupational safety ontology. Section 8 discusses the expected benefits of WA results, while conclusions are reported in Section 9

\section{Framing the Approach}

The WA tool can be classified as a SelfManagement Occupational Safety and Health Supervision System (SMOSHS System Noe et al. (2017)), and as such it empowers its users to early detect risks for her/his safety and health by monitoring the individuals' health status and keeping track of risk exposures and health conditions. The design of the WA tool is based on a multidisciplinary effort among Data Scientists, Sociologists, Psychologists and Occupational and Health specialists towards the development of a User Data Model of working behaviors. The following concepts are the core of the research and the technological implementation of the project.

Protection and Prevention. State the risks associated with each specific working settings, the consequent injuries and health diseases and the actions that can be put in place to prevent them.

Health and Well-being Monitoring. Determine and assess the users' physical and emotional statuses during their working routine. Keep into account users' preferences about her/his interactions with the WA tool, to effectively promote proactive actions concerning her/his own well-being.

Extended Capabilities. Investigate the integration into the WA tool of intelligent functionalities to compensate the diminishing cognitive and physical capabilities of users as well as to assist them issuing context-aware adapting feed-backs.

Autonomous Sensing Systems. Design, develop and integrate sensory systems for a comprehensive collection of data related to users' status and the related environmental conditions.

Decision Support System. Develop a reasoning system concerning users' data to automate the computation of key-indicators measuring several figures of merit of the user status able to provide a reliable source of information for both the endusers and possibly also occupational health and/or safety-responsible specialists.

The consortium of the WA project brings together private and public organizations, large and small/medium enterprises with the common aim of introducing an industry-grade product, i.e., the WA tool, designed on the above listed concepts and employing solutions based on off-the-shelf devices and technologies that can be effectively validated in several case studies. The user interface of the WA tool is based primarily on voice, gesturing and in-site Augmented Reality. How- 


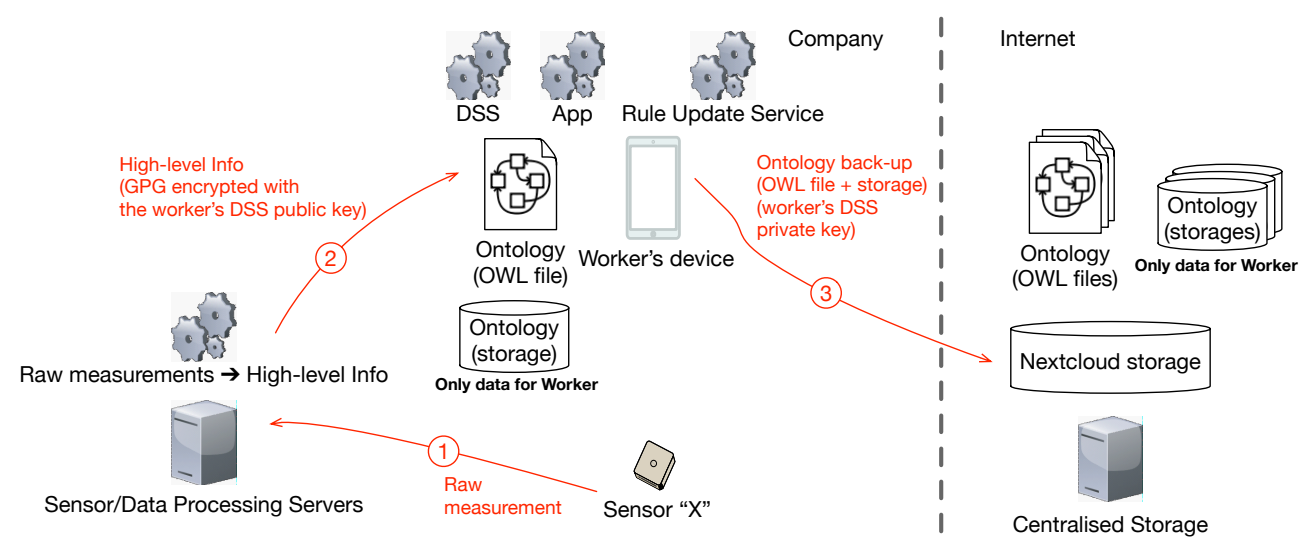

Fig. 1. Architecture of the WA tool

ever, the user will have flexibility to adapt the interface of the system according to her/his preferences with a clear understanding of the advantages as well as of the ethics, privacy, security and regulatory implications entailed by the use of the tool [Collins et al. (2019); Bhave et al. (2020)].

The analyses on the ergonomics of the sensors and on the effectiveness of the user interface of the WA tool as well as of the data collection and processing performed by the WA tool are validated in three actual working and living environments, namely office, manufacturing, and driving, considering 30 workers aged over 50 years old for each of them. Security, privacy, and ethical issues are also a focus of the project in such a sensitive environment.

\subsection{Architecture of the WA tool}

Fig. 1 shows the architecture of the WA tool. The measurements collected by environmental sensors and by sensors wore by users are transmitted via a private WiFi network to a set of servers deployed at the company facility running distinct signal processing algorithms for each kind of sensor, which in turn derive a set of high-level information (e.g., from voice recording, an emotion can be derived) related to the each user. Given a worker, the highlevel information extracted from the signal of one of the sensors is sent to a WA application running on her/his smartphone via a secure connection, while the raw data on the servers deployed in the company facility are safely deleted just after the transfer of the high-level information. The WA application manages a small database with the high-level information coming from each kind of sensor and the ontology describing the concepts related to the user's status. The DSS-reasoner is a component of the WA application accessing the said database and ontology to provide its results to the software component managing data visualization and feed-backs to the user. The Rule Update
Service is a further component of the WA application that periodically updates the rules that control the DSS-reasoner depending on the statistics computed on data collected by the system. In this way the logic of the DSS can be adapted to the profile of the user as it changes over time. Currently, the design choice is to set the initial configuration of the DSS (on each user's smartphone) with a list of human-generated rules and to allow the Rule Update Service to automatically update them over time, depending on the data collected from the worker. The specific adaptation mechanism is still under design as it will depend mainly on the types of rules that will be managed (see Section 7.2). Finally, the Centralised Storage acts as a (encrypted) remote backup for the ontology of each user, as described in Section 6

\section{Stress and Strain Assessment}

In [Rohmert (1984)], the author differentiates between physical and psychological strain. Physical strain describes the effects of stress on the muscle and cardiovascular system. Strain reactions resulting from physical exertion manifest themselves in measurable changes of the human body. In contrast, psychological strain refers to the entirety of all detectable influences that have a psychological effect on the working person [Schlick et al. (2018)]. Psycho-social factors such as age identity, work centrality and perceived social support to improve job satisfaction, commitment and motivation should be taken into account. Psychological stress factors or repetitive work processes can be difficult to eliminate and, in the case of psychological problems, can be difficult to treat. These may be alleviated by broader general health maintenance or the development of appropriate coping strategies.

From an ergonomic point of view, there are not only psychological factors, but also physical factors that affect a worker. Physical factors, such 
as musculoskeletal disorders, increase the risk of incapacity and loss of productivity at work. In 2016, 7.4\% of the EU population suffered from one or more work-related health problems [Eurostat Statistics (2016)]. Therefore, strain is an essential aspect for assessing the effects of work on the working person and it plays an important role in the assessment of human work; hence, the reduction of strain is often an essential goal in work structure [Kirchner (1986)].

There is a difference between stress and strain, which is also reflected in occupational health and safety concepts. In the context of ergonomics, stress describes the external characteristics of a work situation that influences the working person. These include, for example, physical and organisational working conditions. Some external factors that represent stress are the same for all people, but the respective impact, the individual's exposure, can be different and change with age. Strain, on the other hand, describes the reactions of the working person to these conditions. Strain is the individual's internal perception of the stressors surrounding him or her. So while external factors that lead to stress are often taken into account in the design of workplaces, the burden on the individual is much more difficult to assess.

Data collected from sensors monitoring the cardiovascular system, the electrodermal activity, the cerebral activity, the voice, the gaze and the environmental noise will be used to model the user's psychological, emotional and health state and improve it over time. The diversity of information coming from different sensors will allow to develop and test data fusion practices to accurately assess the strain condition of the individual worker.

\section{Environmental Monitoring}

The working environment heavily affects worker's physical and psychological conditions. For this reason, WA deploys specific sensors for noise, light, and temperature/humidity. Light and temperature/humidity will be measured using off-theshelf sensors, while for noise the WA approach will rely on a solution employing off-the-shelf components (e.g., a Raspberry PI based device). Noise exposure at work is counted as one of the main sources that (i) can induce stress and increase heart disease rate in the workers [Münzel et al. (2014)]; (ii) can cause hearing loss in the long term [Girard et al. (2009)], and (iii) can cause musculoskeletal disorder due to postural invariance [Evans and Johnson (2000)]. Moreover, listening to music is one of the main inducers that can regulate emotions [Sagha et al. (2015)]. According to the Noise Directive 2003/10/EC developed by European Parliament and Council (2003), noise level of more than $87 \mathrm{~dB}(\mathrm{~A})$ and a peak sound pressure of $200 \mathrm{~Pa}$, should be avoided and whenever noise level between $80 \mathrm{~dB}(\mathrm{~A})$ and $85 \mathrm{~dB}(\mathrm{~A})$ are reached, appropriate measures are necessary.

In the WA framework, the goal is to measure acoustic features at work, and set a warning/recommendation system to notify the workers for a preventive action (e.g., wearing a headphone), if necessary. Along with the noise level, other features can also be monitored from the acoustics, such as the availability of voice (one or more person are talking), laughter or music, as well as abrupt high pressure sounds (e.g., glass breaking, hammering). These high-level acoustic features will be extracted at real-time using OpenSMILE toolbox [Eyben et al. (2010)]. Periodically, statistics on these measures are sent to the user's device to allow the WA application to provide a recommendation.

\section{Sensing of Worker Behavior and State}

This section describes how the set of multimodal behavioral and biosignal data will be collected from an end-user and analysed to allow the WA application to issue the most appropriate action to support the well-being of the worker.

\subsection{Behavioral data acquisition and analyses}

The behavior of a worker is captured with the following sensors.

Facial Expression. Understanding facial expression is very important as it can reveal emotional states and intentions of people. Facial affect analysis can be performed in terms of prediction of expressions, valence and arousal of the expression and the detection of activated facial Action Units (AU) [Sariyanidi et al. (2015)]. Within the WA project, these analyses will be achieved in realtime by focusing on the design and evaluation of a number of Deep Learning (DL) based frameworks starting with a modified version of [Jang et al. (2019)]. The envisaged framework for facial affect analysis exhibits one camera to capture the frontal face, while a second camera will be potentially used to capture the face profile. The frame streams will be fed into the trained models for face detection and facial affect analysis. The analysis results will be integrated with other sensors' outputs to provide a comprehensive and contextualised understanding of the user affective states. Although here we illustrate the analysis of facial affect, other behavioral and biosignal sensor sub-systems are identical to this in terms of framework setup and design.

Voice. Voice is another important indicator of the state of a speaker/user. By processing speech and voice, not only the machine can recognize the content of the speech and extract its positivity 
or negativity, but also the affect the speaker conveys by the way she/he talks (e.g., angry, happy, etc.) The analysis and recognition of speaker states will be achieved through real-time feature extraction and Deep-Learning based classification techniques, and will be used for decision making and deployment of the most appropriate system recommendations.

Body Pose. Body pose provides useful information for monitoring ergonomic habits. For the WA-tool, body posture estimation methods based on 2D images supply the body pose of the user as the location of the main joints and body parts such as head, shoulders, knees, hips. Joint locations can be expressed as image coordinates measured in pixels or world coordinates (3D coordinates) in order to assess the ergonomics aspects that will be used for analysis and deployment of the most appropriate system recommendations.

Gesture. In the WA Tool, the software and infrastructure enabling minimal gesture interaction with the system will be developed. For the interaction, we use off-the-shelf devices utilising advanced image processing and machine vision algorithms to track the users hand gesture and then convert them into commands. These commands will be used as alternative means of interaction with the WA tool when other input modalities may not be used as effectively.

\subsection{Biosignal data acquisition and analyses}

The WA tool will collect specific biosignals to estimate at least three mental and cognitive states:

- the Mental Workload, representing the amount of cognitive resources allocated to perform a certain task. The Mental Workload index is computed as the ratio between the Electro Encephalographic (EEG) Frontal Theta and the EEG Parietal Alpha [Aricò et al. (2015)];

- the Stress, which is strictly correlated with the variation of the tonic component of the GSR signal, in particular with the Skin Conductance Level [Aricò et al. (2016)];

- the Emotional State, which is evaluated by matching the Galvanic Skin Response and the Electrocardiographic signals [Aricò et al. (2017)].

The Mental Workload is evaluated analysing the EEG signal. The EEG is one of the most common and used techniques of neuroimaging, which records human brain electrical activity through electrodes placed on the scalp. The Mental Workload index is evaluated by matching the EEG feature with Electro Oculogram (EOG) feature, in particular with the eye blinks occurrences. The EOG is derived from the polarization potential that is generated within the eyeball by the metabolically active retinal epithelium. The EOG signal can be acquired through electrodes placed over the eyes.

The Stress index and the Emotional states will be evaluated via Electrocardiogram (ECG) and Galvanic Skin Response (GSR) measurements. The ECG measurements will be based mainly on two features: i) the Heart Rate (HRV) and ii) the Heart Rate Variability (HRV), which is commonly used as a quantitative marker describing the activity of the autonomic nervous system during stress. The GSR will allow us to estimate the Electrodermal Activity (EDA), which is a well-accepted indicator of reticular activation and, therefore, of emotion and cognition. When an individual is under mental stress, sweat gland activity is activated and increases skin conductance.

Most of the current smart bracelets allow also to collect, simultaneously and in a non-invasive way, several signals (i.e., ECG, GSR), which will help the WA Tool to monitor health and strain states of the worker.

Eye Tracking (ET). Various ocular measures have been investigated to measure mental workload, such as pupillary changes during mental activity and the number of eye blinks during mental effort. Eye tracking data can be used to detect mental strain, for instance based on repetitive eye movements. These can be measured by saccade, fixation duration, frequency with which a gaze object is fixed as well as the sequence of fixations on different objects.

\section{Data Management and Security}

In WA, the different experimental phases need that the partners involved in the data collection apply a procedure compliant with the General Data Protection Regulation (GDPR) [Merrick and Ryan (2019)]. WA has approached the problem in the following way. Once data are collected from each sensor, the same partner responsible for its deployment pseudonymizes the raw data series removing the identifiable information of the user and, by means of the applications running in the in-company servers, computes the related highlevel features of interest. Subsequently, the same partner encrypts the pseudonymized raw data series using an hybrid public-key/symmetric key encryption scheme and upload the payload to the centralized data storage for backup and sharingamong-partners purposes. The mechanism put in place for uploading and fetching data from the centralized server ensures a cryptographically enforced access control that makes the remotely stored data properly anonymized according to the GDPR. Indeed, no unauthorized partner will be able to decrypt data not intended to be shared with him, and no external entity will be able to access the cleartext content of the repository without the knowledge of the corresponding cryp- 


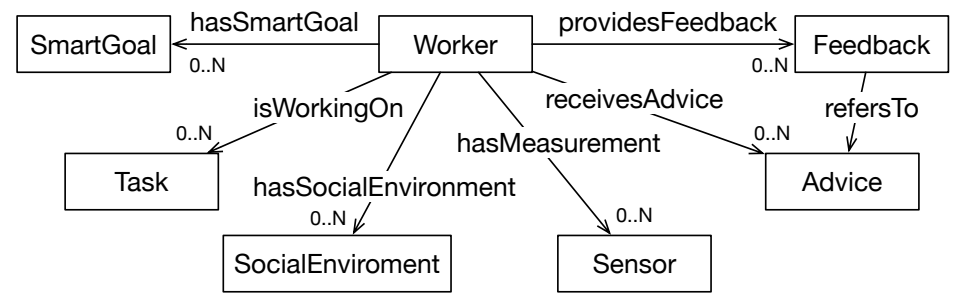

Fig. 2. The WA Ontology main structure.

tographic keys. Finally, the high-level features are encrypted using a hybrid cryptoscheme with the public-key of the WA-agent running on the user's smartphone, that in turn will retrieve the encrypted payload from the in-company servers, periodically.

Data management and privacy will particularly affect the test phases of WA. In fact, the project includes two experimental phases: In-Lab tests and In-Company tests. During the former, controlled environment tests will be conducted; the main objective will be the tuning of the sensors to properly set their accuracy and the equipment selection. The In-Company tests are divided into three sub-phases: Single tests, Week tests and Long-term tests. In each sub-phase, the final WAtool will be tested in real working environments on real users' data, that must be compliant to the rules for individual data protection included in the GDPR. Each partner involved in the experimental data collection and processing will play as Data Processor and will be responsible for such data, while a data sharing agreement among partners will determine the purposes and means for sharing among different partners different subsets of data, and will exhibit a joint Data Controller entity to the end-users (e.g., on the GDPR compliant consent forms). To improve the communication and the interaction among the partners a Local Data Manager has been defined for each of them.

\section{The WA Decision Support System}

The WA Tool provides advices to the users by means of a DSS based on extended logic rules on the content of a KB defined as an OWL ogy. We adopt a model-driven approach, for two reasons: lack of data for training a good model, and availability of good experts who provide their experience for defining both the WA Ontology and the logic rules.

\subsection{The WA Ontology}

The WA Ontology is divided into several Classes, describing workers and their environments (see

\footnotetext{
a The Web Ontology Language (OWL) is a W3C specification and is part of the W3C's Semantic Web technology stack; see: https://www.w3.org/owL/
}

\section{Figure 2,}

Worker.This Class is the "core" of the WA Ontology, ans is defined with some basic Attributes, like Name and Gender. The Attribute Health contains a general description of the worker's health condition. Worker is connected to Task, Sensor, SocialEnvironment, which describe information we are going to collect at run time, about the worker, and Advice and Feedback, which describe advices generated by the DSS and the worker's reactions to them.

Task. This Class describes basic pieces of information about the job currently performed by a Worker. A Task is described by the Structure (i.e., how strictly regulated it is) and Repetitiveness attributes. Note the Timestamp and IsTheLastMeasure Attributes, which permit to extract a history of jobs associated to a give worker, as well as the current job (which is part of the current state of a worker.)

Four sub-Classes have been defined so far: Office, Assembly, Driving and Home; each with specific Attributes. The Home Class refers to home activities, as the WA project aims at considering the whole daily life of the worker.

Sensor. This Class if the root of the most complex taxonomy in the WA Ontology. Here, we describe information we can derive from sensor measurements, about a Worker.

Sensors are divided into four groups: i) Sensors about the environment; ii) Sensors about worker's body and voice; iii) User location; iv) Other sensors. Each provides the Timestamp and IsTheLastMeasure Attributes, which permit to extract a history of measures associated to a given worker, as well as the current measure (which is another part of the current state of a worker). Moreover, sensor measure are associated with a Probability value. Finally, each sensor typology provides its own specific attributes.

The Class Environmentalsensor is the root of sensors collecting environment data, such as temperature, light, noise, etc.

The Class BodySensor is the root of wearable sensors, which collect a vast variety of data, from wearable biometric devices (e.g., ECG and GSR), cameras (e.g., body posture, facial expres- 
sion and eye movements), and microphones (voice recording). For example, from voice recording, we can derive the emotional status of the speaker.

The Class UserLocation is a placeholder for positioning information coming from GPS and/or indoor positioning systems.

The Smartband sensor is a placeholder representing information to collect at the worker's home. We plan to collect information about the wake/sleep rhythm. Further considerations will take privacy into account.

Finally, the Class OtherSensor is currently used to represent questionnaires that will be regularly administered to workers, as a special case of "sensors".

SocialEnvironment. This Class deals with the organisational and social characteristics of the working environment. We expect such characteristics to vary over time, so SocialEnvironment, the Timestamp and Is TheLastMeasure Attributes permit to extract a history of measures associated to a given worker, as well as the current measure (which is another part of the current state of a worker.)

Advice and Feedback. These Classes, which are connected each other, encapsulate the Advice (with Attributes Probability and Typology) generated by the DSS to a given Worker and hes/his Feedback (with Attribute typology) to it.

\subsection{Reasoning}

A set of rules is used to perform reasoning and derive the advices for workers. We opted for the ProbLog [Fierens et al. (2015)] language for our reasoner, because it can deal with probabilistic reasoning, which is interesting as some of the measurements we are going to extract from sensors are probabilistic. We have chosen to describe our KB as an OWL-based Ontology, since it is a standard for Ontologies, and has very useful tools supporting authoring of OWL-bases Ontologies. In particular, we use Protégé.

\section{Expected Benefits}

The expected benefits for workers consist in preventing work-related illnesses, working accidents and stress, symptoms of pandemic illness that impact on older persons more heavily. This by providing a smart environment enriched in comfort and in surveillance thanks to networked sensors. The WA Tool is interesting for insurance companies, as its inclusion in the enterprise information systems can reduce the health insurance costs, through continuous monitoring of health and the possibilities of reacting faster to abnormalities.

Moreover, we can provide arguments to policy makers regarding how to adapt existing regulations (which currently do not differ between the elderly and the young/adult worker) in terms of working conditions, such as working hours, health precautions, retirement age, work and health difficulty.

The benefits concern also Public Health Systems. In fact, work conditions can be consistently rated considering the top sources of stress among individuals which contribute to psychological (e.g., depression, anxiety), physical (e.g., cardiovascular disease, obesity) and behavioral (e.g., increased alcohol abuse, addictions) problems. By ensuring that older workers can remain associated with their companies, the approach can help retain continuous knowledge about the work marketplace, while supporting an ageing population with various employment options. The WA Tool is being set up for different markets w.r.t. the many legal requirements (national and international). All normative and standards are taken into account, with focus on accessibility and interfaces.

\section{Concluding Remarks}

The WA project aims at providing workers with assistance in their everyday routine in the form of recommendations, risks avoidance and reminders. WA will test and validate an integrated solution that will learn the user's behaviour, health data and preferences and, through continue data collection and analysis, will interact naturally with the user. This innovative system will provide workers with assistance in the form of reminders, risks avoidance, and recommendations. In this way, WA will create a scalable product that will empower their user's easing their life by attenuating the impact of aging in their autonomy, work conditions, health and well-being. The paper has described the project aims, the WA Tool architecture, and the ontology for Occupational Safety. At the time of writing this paper, the project is under active development and In-Lab tests have begun. InCompany tests (lasting one year and involving three distinct companies) will collect data and will validate the effectiveness and the efficiency of the adopted technical solutions.

\section{Acknowledgements}

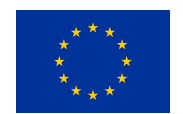

This work was supported by the European Union's Horizon 2020 research and innovation programme under grant agreement No. 826232, project WorkingAge (Smart Working environments for all Ages).

\section{References}

Akkermans, J., A. H. de Lange, B. I. van der Heijden, D. T. Kooij, P. G. Jansen, and J. S. Dikkers (2016). What about time? examining 
chronological and subjective age and their relation to work motivation. Career Dev. Int. 21(4). Aricò, P., G. Borghini, G. Di Flumeri, et al. (2016). Adaptive automation triggered by eegbased mental workload index: a passive braincomputer interface application in realistic air traffic control environment. Frontiers in Human Neuroscience 10, 539.

Aricò, P., G. Borghini, G. Di Flumeri, et al. (2017). Passive BCI in operational environments: insights, recent advances, and future trends. IEEE Transactions on Biomedical Engineering 64(7), 1431-1436.

Aricò, P., G. Borghini, I. Graziani, et al. (2015). Air-traffic-controllers (ATCO): neurophysiological analysis of training and workload. Italian Journal of Aerospace Medicine (12), 35.

Bhave, D. P., L. H. Teo, and R. S. Dalal (2020). Privacy at work: A review and a research agenda for a contested terrain. $J$. Manage. 46(1), 127-164.

Collins, H., K. Ewing, and A. McColgan (2019). Labour law. Cambridge University Press.

De Vroom, B. and E. Øverbye (2017). Ageing and the transition to retirement: A comparative analysis of European welfare states. Routledge.

Dikkers, J., A. De Lange, and B. Van der Heijden (2017). An integrative psychological perspective on (successful) ageing at work. In The Palgrave Handbook of Age Diversity and Work, pp. 67-88. Springer.

European Parliament and Council (2003). Directive 2003/10/EC on the minimum health and safety requirements regarding the exposure of workers to the risks arising from physical agents (noise). Official J. Euro. Union ( $L$ 42) 46

Eurostat

Statistics (2016). Overweight and obesity BMI statistics. Available online: ec. europa.eu/ eurostat/statistics-explained.

Evans, G. W. and D. Johnson (2000). Stress and open-office noise. J. Appl. Psychol. 85(5), 779.

Eyben, F., M. Wöllmer, and B. Schuller (2010). openSMILE: the Munich versatile and fast open-source audio feature extractor. In Proc. 18th Int. Conf. on Multimedia. ACM.

Fierens, D., G. V. den Broeck, J. Renkens, D. Shterionov, I. T. B. Gutmann, G. Janssens, and L. D. Raedt (2015). Inference and learning in probabilistic logic programs using weighted boolean formulas. Theor. Pract. Log. Prog. 15(3), 358-401.

Fitzpatrick, M. D. and T. J. Moore (2018). The mortality effects of retirement: Evidence from social security eligibility at age 62. J. Public Econ. 157, 121-137.

Girard, S. A., M. Picard, A. C. Davis, M. Simard,
R. Larocque, T. Leroux, and F. Turcotte (2009). Multiple work-related accidents: tracing the role of hearing status and noise exposure. $O c$ cup. Environ. Med. 66(5), 319-324.

Goh, J., J. Pfeffer, S. A. Zenios, and S. Rajpal (2015). Workplace stressors \& health outcomes: Health policy for the workplace. Behav. Sci. \& Policy 1(1), 43-52.

Grosch, J. W. and J. C. Scholl (2020). Designing age-friendly workplaces: An occupational health perspective. In Current and Emerging Trends in Aging and Work, pp. 429-451. Springer.

Jang, Y., H. Gunes, and I. Patras (2019). Registration-free face-ssd: Single shot analysis of smiles, facial attributes, and affect in the wild. Comput. Vis. Image Und. 182, 17-29.

Kirchner,

J.-H. (1986). Belastungen und beanspruchungen-einige begriffliche klärungen zum belastungs-beanspruchungs-konzept. In Arbeitsorganisation und Neue Technologien, pp. 553569. Springer.

Merrick, R. and S. Ryan (2019). Data privacy governance in the age of GDPR. Risk Management 66(3), 38-43.

Münzel, T., T. Gori, W. Babisch, and M. Basner (2014). Cardiovascular effects of environmental noise exposure. Eur. Heart J. 35(13).

Noe, R. A., J. R. Hollenbeck, B. Gerhart, and P. M. Wright (2017). Human resource management: Gaining a competitive advantage. McGraw-Hill Education New York, NY.

Rohmert, W. (1984). Das belastungsbeanspruchungs-konzept. Zeitschrift für Arbeitswissenschaft 38(4), 193-200.

Sagha, H., E. Coutinho, and B. Schuller (2015). Exploring the importance of individual differences to the automatic estimation of emotions induced by music. In Proc. 5th Int. Workshop on AVEC, pp. 57-63. ACM.

Sariyanidi, E., H. Gunes, and A. Cavallaro (2015). Automatic analysis of facial affect: A survey of registration, representation, and recognition. IEEE T. Pattern Anal. 37(6), 1113-1133.

Schlick, C., R. Bruder, and H. Luczak (2018). Arbeitswissenschaft. Springer-Verlag.

Surendran, S. (2017). Management accountability for occupational health and safety. Asian J. Manage. 8(4), 1393-1396.

Teimourikia, M. and M. Fugini (2017). Ontology development for run-time safety management methodology in smart work environments using ambient knowledge. Future Gener. Comput. Syst. 68, 428-441.

Tesch-Römer, C. and H.-W. Wahl (2017). Toward a more comprehensive concept of successful aging: disability and care needs. J. Gerontol. B-Psychol 72(2), 310-318. 